\title{
Adaptive Finite Elements with large aspect ratio for aluminium electrolysis
}

\author{
P. Passelli*, M. Picasso ${ }^{\dagger}$ \\ * École polytechnique fédérale de Lausanne \\ Institute of Mathematics, 1015 Lausanne, Switzerland \\ e-mail: paride.passelli@epfl.ch, web page: https://people.epfl.ch/paride.passelli \\ † École polytechnique fédérale de Lausanne \\ Institute of Mathematics, 1015 Lausanne, Switzerland \\ e-mail: marco.picasso@epfl.ch,web page: https://people.epfl.ch/marco.picasso \\ This work is financially supported by Rio Tinto-Aluminium.
}

\begin{abstract}
Aluminium electrolysis is a multiphysics problem (heat and fluid flow, electromagnetism, chemistry, ...) which involves some multi-scale features (from meters to millimeters). A trade-off between computational accuracy and efficiency can then be obtained using adaptive finite elements with large aspect ratio.

The adaptive criteria is based on a so called error indicator justified in [1] for Laplace equation and in [2] for Stokes equation. We construct an adapted mesh such that the error indicator is close to a prescribed tolerance and may have large aspect ratio (up to 100).

Numerical results for Laplace and Stokes equations on flat domains will be first presented and the effectivity index will be studied. An application to aluminium electrolysis for the non linear Navier-Stokes equations with turbulent viscosity will then be discussed.
\end{abstract}

\section{REFERENCES}

[1] M. Picasso Adaptive finite elements with large aspect ratio based on an anisotropic error estimator involving first order derivatives. Computer Methods in Applied Mechanics and Engineering, Vol. 196, pp. 14-23, 2006.

[2] M. Picasso An adaptive algorithm for the Stokes problem using continuous, piecewise linear stabilized finite elements and meshes with high aspect ratio. Applied Numerical Mathematics, Vol. 54, Issues 3-4, pp. 470-490, 2005. 